\title{
Eficiencia biológica de biotipos lecheros de primera lactancia en sistemas a pastoreo
}

\author{
Marini, P.R. ${ }^{1}$; Castro, R. ${ }^{1}$; Frana, E. ${ }^{1}$; Di Masso, R.J. ${ }^{1,2}$ \\ ${ }^{1}$ Facultad de Ciencias Veterinarias, Universidad Nacional de Rosario (UNR), Ovidio Lagos y Ruta 33, \\ Casilda (2170), Santa Fe (Argentina). ${ }^{2}$ Consejo de Investigaciones (CIUNR). \\ E-mail: pmarini@fveter.unr.edu.ar
}

\begin{abstract}
Resumen
Marini, P.R.; Castro, R.; Frana, E.; Di Masso, R.J.: Eficiencia biológica de biotipos lecheros de primera lactancia en sistemas a pastoreo. Rev. vet. 26: 2, 136-142, 2015. El objetivo del trabajo fue evaluar la eficiencia biológica de vacas lecheras de diferentes biotipos en sistemas a pastoreo. Se utilizaron los datos de 271 vacas de primera lactancia recolectados entre los años 1992-2012 en un tambo de Santa Fe (Argentina). Se trabajó con datos retrospectivos de vacas puras $(n=106)$ y vacas con registro de cría $(n=165)$. Se consideraron las variables: edad al primer parto en días (EPP), intervalo primer parto-segunda concepción en días (IPC), producción de leche ajustada a 305 días de lactancia en litros (PL) y cantidad de grasa butirosa producida en $\mathrm{kg}(\mathrm{GB})$. Estadísticamente se emplearon análisis univariados (test "t" de Student) y multivariados (componentes principales). Se registraron diferencias significativas para los caracteres productivos PL y GB. Las vacas puras produjeron 478 litros más de leche y $40 \mathrm{~kg}$ más de grasa. Las vacas de uno y otro grupo no se diferenciaron significativamente ni en la edad al primer parto (que tendió a ser mayor en las no-puras: $+19,5$ días) ni en el intervalo parto-concepción (que tendió a ser mayor en las puras: 15,5 días). Los dos primeros componentes principales explicaron el $75,25 \%$ de la variancia total observada. El primer componente explicó el 44,60\% de la variancia y se correlacionó en forma negativa y significativa con la producción de leche (PL: $\mathrm{r}=-0,836)$ y de grasa (GB: $\mathrm{r}=-0,939)$. El segundo componente explicó el $30,65 \%$ de la variancia y se correlacionó en forma negativa y significativa con la edad al primer parto (EPP: $\mathrm{r}=-0,795$ ) y en forma positiva con el intervalo parto-concepción (IPC: $\mathrm{r}=-0,674)$. Se concluye que los biotipos estudiados se comportan de manera distinta entre ellos, aunque coinciden en que las vacas que tardan más tiempo en tener su primer parto, responden productiva y reproductivamente de manera más conveniente que las vacas que paren antes su primer ternero independientemente de su biotipo de origen.
\end{abstract}

Palabras clave: vaca lechera, primera lactancia, eficiencia, sistema a pastoreo.

\begin{abstract}
Marini, P.R.; Castro, R.; Frana, E.; Di Masso, R.J.: Biological efficiency of first lactation dairy biotypes on grazing systems. Rev. vet. 26: 2, 136-142, 2015. The aim of this study was to evaluate the biological efficiency of dairy cows of different biotypes in grazing systems. The data of 271 first lactation cows collected between 1992-2012 in Santa Fe (Argentina), were used. Retrospective data of dairy cows, divided into two categories: pure cows $(\mathrm{n}=106)$ and record breeding cows $(\mathrm{n}=165)$, were employed. Considered variables were: age at first calving (in days, EPP), interval between first lactation second conception (in days, IPC), milk production adjusted to 305 days of lactation (in liters, PL) and amount of butyrous fat produced (in kg, GB). Univariate techniques (Student "t" test) and multivariate system (principal components) were considered for statistical analysis. Significant differences between PL and GB were registered. Pure cows produced more milk (478 liters) and more fat (40 kg). No statistical differences were found between both groups regarding age at first lactation (which tended to be higher for not pure cows: +19.5 days) or calving conception interval (which tended to be higher for pure cows: 15.5 days). The first two principal components accounted for $75.25 \%$ of the total observed variance. The first component explained $44.60 \%$ of variance and correlated negatively and significantly with milk production (PL: $\mathrm{r}=-0.836$ ) and fat production (GB: $r=-0.939$ ) and second component explained $30.65 \%$ of variance and cor-
\end{abstract}


related negatively and significantly with age at first calving (EPP: $r=-0.795$ ) and positively with calving conception interval (IPC: $r=-0.674$ ). In conclusion, the studied biotypes behave differently between them, though they agree that cows took longer to have their first lactation, respond productive and reproductively higher so that the cows that calved before their first calf, regardless its original biotype.

Key words: dairy cow, first lactation, efficiency, grazing system.

\section{INTRODUCCIÓN}

La vaca lechera actual, de alto rendimiento, ha sido formada biológicamente acorde a las demandas del mercado mediante la aplicación de una presión selectiva, continua, des-balanceada y descuidada, que la ha acercado al límite de su potencial vital. Cada vez se hace más difícil suministrarle un medio ambiente nolimitante, siendo casi imposible lograr este propósito durante la fase inicial de su lactancia.

El notable incremento en el desempeño productivo y el tamaño de estas vacas modernas de alta producción, ha sido posible por el uso reiterado y asimétrico de una selección basada exclusivamente en la producción de leche. Si bien este proceso fue acompañado de modificaciones del ambiente nutricional, las mismas han sido insuficientes para evitar el deterioro de funciones vitales tales como la reproducción y la supervivencia ${ }^{2}$.

En diferentes países se han diseñado numerosas estrategias productivas que combinan distintos ambientes con diversos grupos genéticos, dando lugar a una amplia y variada gama de sistemas de producción, con distintos resultados tanto productivos como reproductivos. En el caso de Argentina la producción de leche se basa principalmente en el uso de pasturas y verdeos suplementados con granos y forrajes conservados ${ }^{4}$.

La edad al primer parto (EPP) es un indicador del tiempo que tarda un animal en alcanzar su madurez sexual y reproducirse por primera vez ${ }^{9}$. Como tal, refleja diferencias en la velocidad de crecimiento y en la edad a la pubertad de las hembras. Una pubertad tardía reduce el valor económico del animal al disminuir el número potencial de descendientes y de lactancias producidos en su vida útil ${ }^{8}$.

La EPP puede verse afectada por el tamaño corporal del animal y por el inicio de la actividad hormonal del sistema reproductivo ${ }^{14}$. Se considera que la edad a la pubertad no está solamente determinada por un peso en particular, sino por un conjunto no bien determinado de condiciones fisiológicas que resultan de un peso dado ${ }^{8}$. Si bien una pubertad temprana sería un rasgo deseable, no existe acuerdo al respecto en la bibliografía, en tanto algunos autores han hecho notar que un parto temprano afecta negativamente la producción de leche y la longevidad ${ }^{1,6,15,18,20}$.

El objetivo de este trabajo fue evaluar la eficiencia biológica de vacas lecheras de diferentes biotipos, de primera lactancia, en sistemas a pastoreo.

\section{MATERIAL Y MÉTODOS}

Se utilizaron los datos de 271 vacas de primera lactancia recolectados entre los años 1992-2012 en el tambo cabaña Holando Argentino perteneciente a la Escuela Agrotécnica General San Martín dependiente de la Universidad Nacional de Rosario. El mismo se encuentra ubicado en la localidad de Casilda, Provincia de Santa Fe, Argentina ( $33^{\circ} 02^{\prime} 39^{\prime \prime}$ de latitud sur, $61^{\circ}$ $10^{\prime} 05^{\prime \prime}$ de longitud oeste). El establecimiento cuenta con control lechero oficial de la Sociedad Rural de Totoras (entidad oficial $\mathrm{N}^{\circ} 13$ ). En el lapso relevado todas las vacas se manejaron en las mismas instalaciones de ordeño.

Los datos retrospectivos correspondientes a las lactancias de vacas primíparas de genotipo Holstein Americano-Canadiense con registro completo, se dividieron en dos categorías: vacas puras $(\mathrm{VP}, \mathrm{n}=106)$ y vacas con registro de cría (VRC, $\mathrm{n}=165$ ). La diferencia entre ambas se basa en que las vacas puras se inseminan siempre con semen de toros probados, práctica que no se mantiene de manera constante en el caso de las VRC. Durante el período objeto de esta evaluación las vacas consumieron forrajes bajo pastoreo directo (pasturas polifíticas y verdeos anuales de invierno y de verano) o conservados (silajes de planta entera de maíz y sorgo, henos de praderas) y concentrados (granos de maíz y sorgo).

Durante el período considerado el clima fue muy variable, tanto en precipitaciones como en la combinación de temperatura y humedad relativa ambiente. Las vacas pertenecientes a cada uno de los grupos mencionados se caracterizaron en función de los valores de las siguientes variables: EPP, intervalo primer partosegunda concepción en días (IPC), producción de leche ajustada a 305 días de lactancia en litros (PL) y cantidad de grasa butirosa producida en $\mathrm{kg}(\mathrm{GB})$.

Pese a que la cantidad de grasa producida está relacionada con la cantidad de leche originada, se incluyeron ambas variables productivas porque PL no refiere a los valores reales sino que se la ha ajustado a una duración de la lactancia común a todos los individuos, mientras que GB indica los kg de grasa butirosa efectivamente producidos en la primera lactancia. La inclusión de las variables reproductivas EPP e IPC se justifica porque la primera guarda relación con la edad al primer servicio -y por ende con la precocidad- y la segunda con el impacto que el inicio de la lactancia tiene sobre el reinicio de la actividad reproductiva. 
El efecto del tipo de vaca sobre las diferentes variables mencionadas se evaluó con un análisis univariado utilizando la prueba t de Student para datos independientes. Con el fin de investigar posibles agrupamientos asimilables a potenciales grupos con diferente aporte de las variables evaluadas, se aplicó la técnica multivariada de componentes principales ${ }^{3}$. A los efectos del análisis, los componentes principales generados en el análisis multivariado, correspondientes a cada animal, fueron tratados como nuevas variables aleatorias. Los valores individuales correspondientes al primer ( $\mathrm{PC} 1)$ y segundo componente principal (PC2) se graficaron en un sistema de coordenadas cartesianas ortogonales. La identificaron de los animales ubicados en cada cuadrante permitió definir cuatro grupos de vacas. La asociación entre ambas categorías (tipo de vaca y cuadrante) se evaluó con una prueba $\mathrm{X}^{2}$ de independencia.

El análisis de la variancia a un criterio de clasificación (cuadrante) seguido de la prueba de comparaciones múltiples de Bonferroni, se utilizó para evaluar el efecto del cuadrante de pertenencia sobre cada una de las variables productivas y reproductivas mencionadas, a las que se sumaron dos indicadores vinculados con la longevidad de las mismas: NP (número de partos registrados a lo largo de su vida reproductiva total) e IL (índice de leche, definido como la producción de leche por día de vida de las vacas y calculado como el cociente entre la producción total de leche en litros alcanzada por cada vaca a lo largo de su vida y el número de días requeridos para producirlos).

\section{RESULTADOS}

Análisis univariado. La Tabla 1 resume los valores de los indicadores productivos y reproductivos en ambos grupos de vacas. Se observaron diferencias estadísticamente significativas para los caracteres productivos Pl y GB. En promedio, a lo largo del ciclo estudiado, las vacas puras produjeron 478 litros más de leche y 40 $\mathrm{kg}$ más de grasa. Las vacas de uno y otro grupo no se diferenciaron significativamente ni en la edad al primer parto (que tendió a ser mayor en las no puras: $+19,5$ días) ni en el intervalo parto-concepción (que tendió a ser mayor en las puras: 15,5 días).

Tabla 1. Indicadores productivos y reproductivos en dos tipos de vacas lecheras de primera lactancia.

\begin{tabular}{lcccc}
\hline indicador & VP & VRC & $t$ & $p$ \\
\hline PL (kg) & $5626 \pm 135,6$ & $5148 \pm 84,9$ & 4,463 & $<0,0001$ \\
GB (kg) & $244,6 \pm 7,56$ & $204,5 \pm 5,88$ & 4,215 & $<0,0001$ \\
EPP (días) & $967,0 \pm 16,72$ & $986,5 \pm 15,98$ & 0,8151 & 0,4157 \\
IPC (días) & $207,5 \pm 12,22$ & $192,0 \pm 9,92$ & 0,9775 & 0,3292 \\
\hline
\end{tabular}

PL: producción de leche, GB: producción de grasa butirosa, EPP: edad al primer parto, IPC: intervalo primer parto-concepción, VP: vacas puras, VRC: vacas con registro de cría, t: Student, p: significancia. Tamaño muestral - VP: $n=106$; VRC: $\mathrm{n}=165$. Valores expresados en media aritmética \pm error estándar.
Análisis multivariado. Los dos primeros componentes principales explicaron el $75,25 \%$ de la variancia total observada. El primer componente (PC1) explicó el $44,60 \%$ de la variancia y se correlacionó en forma negativa y significativa con producción de leche (PL: $\mathrm{r}=$ $-0,836)$ y producción de grasa (PG: $r=-0,939)$, en menor medida con el intervalo parto-concepción (IPC: $r=$ $-0,449)$ y no mostró correlación con la edad al primer parto (EPP: $r=0,047)$. Como resultado de estas asociaciones, el primer componente principal se denominó "producción" (a mayor valor del componente menor producción de leche y menor producción de grasa). Los animales de menor producción tendrían, a su vez, un menor intervalo parto-concepción. La producción no se vería afectada por la edad al primer parto.

Dado que las vacas puras tienen mayor producción de leche y mayor producción de grasa de acuerdo a lo concluido en el análisis univariado, le corresponden a las mismas un valor menor de este componente [media aritmética \pm error estándar: $\mathrm{VP}=-0,4823 \pm$ $0,1370 ; \mathrm{VRC}=0,3076 \pm 0,0945 ; \mathrm{t}=4,904 ; \mathrm{p}<0,0001]$. El segundo componente (PC2) explicó el 30,65\% de la variancia y se correlacionó en forma negativa y significativa con la edad al primer parto (EPP: $r=-0,795$ ) $\mathrm{y}$ en forma positiva con el intervalo parto concepción (IPC: $r=-0,674)$, en menor medida con la producción de leche (PL: $r=-0,373$ ) y no mostró correlación con la producción de grasa (GB: $r=-0,030$ ). En consecuencia este componente se denominó "reproducción" (a mayor valor del componente menor edad al primer parto y mayor intervalo parto concepción).

Los animales sexualmente más precoces se preñan antes y paren antes, tienen mayor producción de leche y un mayor intervalo parto-concepción. La producción de grasa no afecta el comportamiento reproductivo (en realidad: el IPC). Pese a que las vacas puras tienen mayor producción de leche, el análisis univariado indicó ausencia de diferencias estadísticamente significativas entre vacas puras y no puras para edad al primer parto e intervalo parto-concepción, por lo que las dos categorías de animales no se diferencian en el valor de la segunda componente [media aritmética \pm error estándar: $\mathrm{VP}=-0,0548 \pm 0,0991 ; \mathrm{VRC}=0,0349 \pm 0,0916 ; \mathrm{t}$ $=0,644 ; p=0,521]$. La Figura 1 presenta la distribución de las 271 vacas en el plano cartesiano definido por los valores de los dos primeros componentes principales del análisis multivariado. Tomando como criterio el origen de coordenadas se definieron cuatro cuadrantes numerados en forma consecutiva en sentido contrario a las agujas del reloj.

Entre los cuadrante surgieron diferencias: cuadrante I (valores positivos de ambas componentes): $\mathrm{PCl}>0$ menor producción de leche y menor producción de grasa y $\mathrm{PC} 2>0$ menor edad al primer parto y mayor intervalo parto-concepción. cuadrante II (valores negativos de $\mathrm{PC} 1$ y positivos de $\mathrm{PC} 2$ ): $\mathrm{PC} 1<0$ mayor 
producción de leche y mayor producción de grasa y PC2 $>0$ menor edad al primer parto y mayor intervalo parto-concepción. cuadrante III (valores negativos de ambas componentes): $\mathrm{PCl}<0$ mayor producción de leche y mayor producción de grasa y $\mathrm{PC} 2<0$ mayor edad al primer parto y menor intervalo parto-concepción. Cuadrante IV (valores positivos de PC1 y negativos de $\mathrm{PC} 2$ ): $\mathrm{PCl}>0$ menor producción de leche y menor producción de grasa y $\mathrm{PC} 2<0$ mayor edad al primer parto y menor intervalo parto-concepción.

Se determinó la proporción de vacas de una y otra categoría incluidas en cada cuadrante. La distribución de los dos tipos de vacas no fue independiente del cuadrante $\left(X^{2}=30,3 ; p<0,0001\right)$. Se observó un predominio de vacas puras en los cuadrantes II (VP: $26 \%$; VRC: $16,5 \%$ ) y III (VP: $38,5 \%$; VRC: $14,7 \%$ ) es decir, en aquellos con valores negativos de PC1 (mayor producción) $\mathrm{y}$, principalmente en el cuadrante III (valores negativos de PC2: peor desempeño reproductivo).

Las vacas VRC, por su parte, predominaron en los cuadrantes I (VP: 18,3\%; VRC: $32,5 \%$ ) y IV (VP: 17,3\%; VRC: $36,2 \%$ ), con valores positivos de PC1 (menor producción) y valores positivos (cuadrante I: mejor desempeño reproductivo) y negativos (cuadrante II: peor desempeño reproductivo) de PC2.

La Figura 2 resume los valores (promedio \pm error estándar) de los indicadores productivos y reproductivos utilizados en el análisis multivariado (productivos: PL y GB; reproductivos: EPP e IPC con el agregado del número de partos (NP) y el índice de leche (IL) como indicadores de longevidad.

\section{DISCUSIÓN}

La producción mundial de vacas lecheras ha aumentado notablemente en los últimos 40 años y, en algunos países europeos, ha duplicado sus niveles. Este cambio ha sido posible gracias a la combinación de mejoras tanto genéticas (selección artificial enfocada hacia la producción individual) como ambientales (alimentación y manejo), todo ello guiado con un enfoque particular que enfatizó el volumen de producción láctea ${ }^{21}$.

La tasa de ganancia genética en producción de leche alcanza el $1,5 \%$ por año, gracias al uso eficaz de la inseminación artificial, a las pruebas de progenie y a la intensa selección de los toros para su uso a nivel mundial. Esta alta productividad se ha visto acompañada de una documentada disminución global de la eficiencia reproductiva de los hatos lecheros, entendida como la capacidad de la hembra de producir un ternero vivo, particularmente en el caso de la raza Holstein, predominante a nivel global ${ }^{24}$.

Este mismo esquema es el que se ha llevado a cabo en países de Latinoamérica. En Argentina, entre los años 2001 y 2011, la producción de semen de origen nacional disminuyó un $18 \%$ mientras que el importado aumentó un 135\%, alcanzando en 2011 un total de 2.123.587 pajuelas, lo que representó el $82 \%$ del mer- cado frente a las 467.854 pajuelas (18\% restante) de semen nacional ${ }^{5}$.

Tal información permite especular que, a través de esta migración de gametas, el progreso genético constatado en los países de origen de los reproductores se trasladaron, en gran medida, al rodeo de vacas Holstein de Argentina, concepto que es compartido en general por importadores, vendedores y asesores. Las consecuencias de dicha aseveración entran en colisión con los propósitos declarados en una visión más totalizadora de la producción lechera nacional que pone énfasis en el ajuste que debe existir entre el potencial genético de una especie productiva y las particularidades del ambiente en el que esa especie debe expresar dicho potencial ${ }^{13}$.

Los resultados obtenidos en este análisis permitieron trabajar con dos grupos de vacas que se diferencian significativamente utilizando el criterio ampliamente difundido de producción de leche ajustada a 305 días de lactancia. Las producciones de leche de ambos grupos coinciden con los datos informados en algunas publicaciones ${ }^{23}$, aunque son menores a las obtenidas por otros a nivel local ${ }^{11}$, con valores entre 6500 litros y 7500 litros para las mismas condiciones de manejo y región. Los grupos no presentaron diferencias significativas en la edad promedio al primer parto. Los valores relevados para estas variables son mayores que los informados por otros autores ${ }^{12,18,22}$.

Tampoco se observaron diferencias significativas en los valores promedio del IPC, siendo los mismos superiores a los informados anteriormente ${ }^{11}$. La consideración conjunta de estas variables estaría indicando que, en promedio, estas vacas no presentan una elevada producción de leche, llegan al primer parto a una edad avanzada y presentan dificultades para quedar preñadas luego del primer parto.

$\mathrm{Al}$ analizar los resultados obtenidos dentro de cada grupo (Figura 1) se puede interpretar que las vacas que están en el cuadrante I se preñaron antes (menor edad

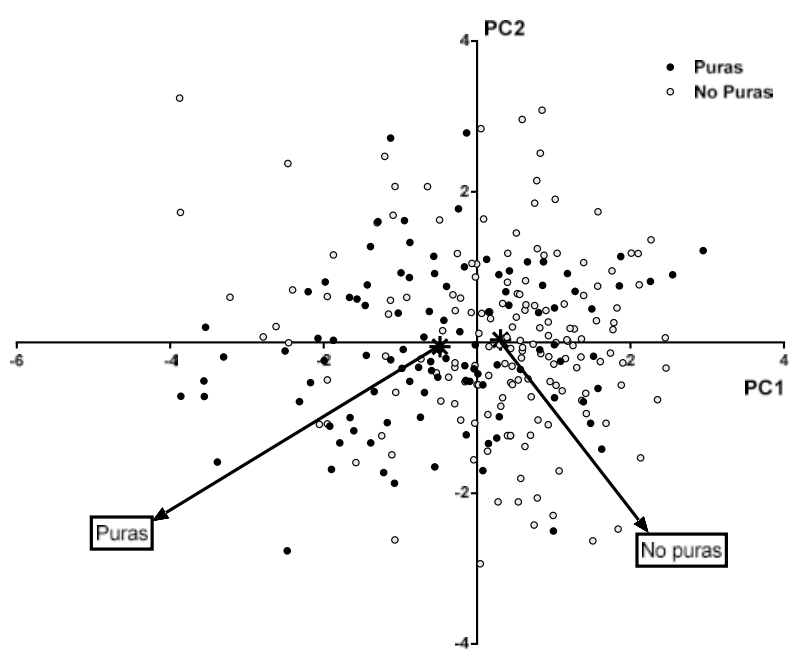

Figura 1. Distribución de las vacas en el plano cartesiano definido por los dos primeros componentes principales $(\mathrm{PC})$. 
al primer parto) sin embargo presentan menor producción y pese a ello tienen mayor intervalo parto-concepción. Podrían denominarse vacas "problemáticas". Las vacas del cuadrante II se preñaron antes (menor edad al primer parto) con alta producción, lo que afecta el intervalo parto-concepción, son vacas "productoras típicas con desadaptación".

Las vacas del cuadrante III se preñaron más tarde (mayor edad al primer parto) con alta producción, sin que ello afecte el intervalo parto-concepción, son vacas "interesantes". Habría que verificar su desempeño posterior en tanto su "alta" producción no afecte su reproducción inmediata. Las vacas del cuadrante IV se preñaron más tarde (mayor edad al primer parto), con menor producción y menor intervalo parto-concepción. Son vacas "para sistemas extensivos sobre pasturas". Se sabe que la producción de leche en pastoreo se basa fundamentalmente en el aprovechamiento del crecimiento de las praderas, usando al forraje como fuente básica de alimentación ${ }^{17}$. La alta competitividad de este sistema está relacionada con el patrón estacional del crecimiento de las pasturas, que depende básicamente de una apropiada humedad y fertilidad del suelo ${ }^{10,16}$.

Las vacas puras predominaron en el cuadrante II que caracteriza a aquellas "productoras típicas con desadaptación" pero también abundaron en el cuadrante III (vacas interesantes). La diferencia radica en que las vacas puras del cuadrante III poseen una mayor edad al primer parto que les permite llegar a su primer parto en mejores condiciones de reservas corporales a su primera lactancia para mantenerse, producir y volver a gestar lo antes posible. Estas vacas llegan en promedio con cinco meses más que las vacas del cuadrante II, indicando que a un promedio de ganancia de $600 \mathrm{~g} / \mathrm{día}$, entrarían a su primer servicio con $90 \mathrm{~kg}$ más de peso vivo.

\begin{tabular}{|c|c|c|c|c|c|c|c|}
\hline $\begin{array}{lll} & \\
\end{array}$ & & $\begin{array}{l}\text { Puras } \\
26 \%\end{array}$ & $\begin{array}{c}\text { No Puras } \\
16,5 \%\end{array}$ & & $\begin{array}{l}\text { Puras } \\
18,3 \%\end{array}$ & $\begin{array}{c}\text { No Puras } \\
32,5 \%\end{array}$ & $\mathrm{Cl}$ \\
\hline & EPP & $870 \pm 138$ a & $870 \pm 107 a$ & EPP & $867 \pm 128$ a & $854 \pm 126 a$ & \\
\hline & IPC & $290 \pm 13$ a & $336 \pm 16 b$ & IPC & $191 \pm 15$ a & $199 \pm 11 b$ & \\
\hline & NP & $3,2 \pm 0,3$ a & $2,7 \pm 0,3 b$ & NP & $3,1 \pm 0,4 \mathrm{a}$ & $4,1 \pm 0,2 \mathrm{~b}$ & \\
\hline & PL & $5694 \pm 173$ a & $5864 \pm 134 b$ & PL & $4144 \pm 207 a$ & $4311 \pm 95 b$ & \\
\hline & GB & $284 \pm 10$ a & $280 \pm 89$ a & GB & $161 \pm 12$ a & $165 \pm 37 a$ & \\
\hline & IL & $5,2 \pm 0,2 \mathrm{a}$ & $5,8 \pm 0,3 b$ & IL & $4,7 \pm 0,3 \mathrm{a}$ & $4,8 \pm 0,2 \mathrm{a}$ & \\
\hline & & $\begin{array}{l}\text { Puras } \\
38,5 \%\end{array}$ & $\begin{array}{c}\text { No Puras } \\
14,7 \%\end{array}$ & & $\begin{array}{l}\text { Puras } \\
17,3 \%\end{array}$ & $\begin{array}{c}\text { No Puras } \\
36,2 \%\end{array}$ & PC1 \\
\hline & EPP & $1031 \pm 125$ a & $1054 \pm 154$ a & EPP & $1099 \pm 195$ a & $1131 \pm 210 a$ & \\
\hline & IPC & $171 \pm 10$ a & $153 \pm 17 b$ & IPC & $111 \pm 16$ a & $116 \pm 10 \mathrm{a}$ & \\
\hline & NP & $3,3 \pm 0,2 \mathrm{a}$ & $3,2 \pm 0,3 a$ & NP & $3,6 \pm 0,4 a$ & $3,8 \pm 0,2 \mathrm{~b}$ & \\
\hline & PL & $7025 \pm 142$ a & $6526 \pm 142 b$ & PL & $5121 \pm 212 a$ & $4911 \pm 90 \mathrm{~b}$ & \\
\hline & GB & $284 \pm 8$ a & $255 \pm 49 b$ & GB & $177 \pm 13$ a & $175 \pm 33$ a & \\
\hline C III & IL & $2,6 \pm 0,2 a$ & $3,2 \pm 0,3 b$ & IL & $2,3 \pm 0,3$ a & $3,8 \pm 0,2 b$ & C IV \\
\hline
\end{tabular}

Figura 2. Caracterización productivo-reproductiva de dos tipos de vacas lecheras en su primera lactancia y a lo largo de su vida útil, discriminadas de acuerdo al cuadrante de pertenencia (C) definido por los valores de las dos primeras componentes (PC) generadas en el análisis de componentes principales.
Esta misma situación se da en el grupo de vacas no puras pertenecientes al cuadrante I (denominadas "problemáticas") en tanto las del cuadrante IV serían las adecuadas para el tipo de sistema a pastoreo de la zona. Un trabajo de la Asociación Argentina de Criadores de Holando sobre 106.464 primeras lactancias cerradas en el período 2000-2005 del control lechero oficial, reveló que la edad al primer parto de vaquillonas fue de 32 meses, ocurriendo el $50 \%$ de los partos entre los 28 y 32 meses ${ }^{23}$

Una opinión generalizada entre productores y profesionales es que la edad óptima al primer parto de vacas lecheras es entre los 22 y 27 meses y con un mínimo de $500 \mathrm{~kg}$ de peso ${ }^{7}$. La edad y el peso al primer servicio fértil tuvieron un efecto significativo sobre la producción de leche en primera lactancia ${ }^{19}$. En este caso, la edad al primer parto no afectó la producción de leche en la primera lactancia pero sí la capacidad de las vaquillonas para reiniciar la actividad reproductiva. El efecto perjudicial de la menor edad al primer parto sobre los indicadores reproductivos no estaría relacionado directamente con la producción en el sentido que indican las correlaciones informadas (a mayor producción, mayores intervalos) sino indirectamente en términos de balance energético (a menor edad, igual producción), implicando desbalance energético.

Sin embargo, mantener esa producción con menor edad afecta negativamente el desempeño reproductivo posterior de la vaca, no tanto la aparición del primer celo sino el logro de una segunda concepción efectiva. Estos resultados coincidirían en parte con los obtenidos por otros investigadores para quienes el balance energético negativo afectaría el restablecimiento de la actividad ovárica y el porcentaje de ovulaciones sin manifestación de celo, profundizado en vacas de primer parto, categoría que debe destinar parte de la energía consumida para completar su desarrollo ${ }^{25}$.

Respecto al análisis entre grupos, al comparar los dos grupos de vacas dentro de cada cuadrante con respecto al comportamiento de las variables analizadas, surge que las VRC del primer cuadrante presentaron mayor producción de leche ajustada a 305 días, lo que se tradujo (posiblemente) en un mayor intervalo parto-concepción en comparación con las VP. No se observaron diferencias entre ambos grupos en la edad al primer parto, en la producción real de grasa butirosa, ni en el índice de leche. Las VRC tuvieron, en promedio, un parto más que las VP.

Las VRC del segundo cuadrante presentaron mayor producción de leche ajustada a 305 días lo que se tradujo (posiblemente) en un mayor intervalo partoconcepción, en comparación con las VP. No se observaron diferencias entre ambos grupos en la edad al primer parto ni en la producción real de grasa butirosa, pero sí 
en el índice de leche, que fue mayor en las VRC. Las VRC tuvieron, en promedio, un menor número de partos que las VP. Las VRC del tercer cuadrante presentaron menor producción de leche ajustada a 305 días lo que se tradujo (posiblemente) en un menor intervalo parto-concepción en comparación con las VP. También presentaron menor producción de grasa butirosa.

No se observaron diferencias entre ambos grupos en la edad al primer parto, tampoco en el número de partos, ni en la producción real de grasa butirosa, pero sí en el índice de leche, que fue mayor en las VRC. Las VRC tuvieron, en promedio, un índice de leche superior al de las VP, y las VRC del cuarto cuadrante presentaron menor producción de leche ajustada a 305 días en comparación con las VP, sin diferencias entre ellas en el intervalo parto-concepción. Los grupos no se diferenciaron en la producción de grasa butirosa. No se observaron diferencias entre ambos grupos en la edad al primer parto, pero sí en el número de partos, que fue algo mayor en las VRC. El índice de leche también fue mayor en las VRC (Figura 2).

Restringiendo la comparación a estos dos cuadrantes, las vacas más adecuadas -si se priorizan aspectos reproductivos sobre productivos- serían las del cuadrante 1 ya que se preñan con una menor edad, presentan valores de intervalo parto-concepción intermedios y son las que mayor longitud de vida productiva tienen, aunque comparativamente, producen menos leche $\mathrm{y}$, por ende, menos grasa. Surge así el interrogante respecto a la causa del mayor intervalo parto-concepción (199 vs 116 días), dado que producen menos leche (4311 vs 4911 litros). Tal vez la respuesta esté dada por su menor edad al primer parto (854 vs 1131 días), que afectaría negativamente el reinicio de la actividad reproductiva luego de su primer parto, postura coincidente con la de otros investigadores del tema ${ }^{25}$.

La mayor cantidad de vacas se ubicaron en los cuadrantes 2 y 3 (C1: más leche). De dichos cuadrantes, las vacas más adecuadas podrían ser las del $\mathrm{C} 2$ ya que se preñan con una menor edad, si bien producen menos y presentan mayores valores de intervalo- parto-concepción y tienen un mayor índice de leche. Se reitera lo argumentado previamente en el sentido que la precocidad (menor edad al primer parto) se asocia con una menor producción y un mayor intervalo parto-concepción. Parte de los resultados coinciden con una tendencia mundial de llegar con una menor edad al primer parto ${ }^{22}$ como lo indican los países con sistemas intensivos, aunque los resultados obtenidos nos permitirían discutir si para los sistemas a pastoreo esa búsqueda de menor edad al primer parto sería eficiente para nuestros sistemas.

Se concluye que los biotipos estudiados se comportan de manera distinta entre ellos, aunque coinciden en que las vacas que tardaron más tiempo en tener su primer parto responden productiva y reproductivamente de manera superior que las vacas que parieron antes su primer ternero, independientemente de su biotipo de origen.

\section{REFERENCIAS}

1. Bormann J, Druet T, Gengler N, Wiggans GR. 2002. Estimating effects of permanent environment, lactation stage, age, and pregnancy on test-day yield. J Dairy Sci 85: 263-284.

2. Camargo O. 2012. La vaca lechera entre la eficiencia económica y la ineficiencia biológica. Arch Zootec 61: 13-29.

3. Carrasco JL, Hernán MA. 1993. Estadística multivariante en las ciencias de la vida. Ed. Ciencia 3, Madrid, $364 \mathrm{p}$.

4. Dutour EJ, Melucci LM. 2010. Asociación entre parámetros productivos y reproductivos de vacas lecheras de acuerdo el sistemas de producción. Rev Arg Prod Anim 18: 133-147.

5. Etcheverry J. 2012. Situación y evolución del mercado de la genética bovina en la Argentina y el mercado internacional. Jornadas $40^{\circ}$ Aniversario CABIA. On line: http//181.119.20.20/cabia/Etcheverry

6. Ettema J, Santos J. 2004. Impact of age at first calving on lactation, reproduction, health, and income in first parity holstein on comemercial farms. J Dairy Sci 87: 2730-2742.

7. Galvis R. 2008. Aspectos fisiológicos del crecimiento con relación a la producción de leche. Publ. Universidad Nacional de Colombia, Medellín, 74 p.

8. Grajales H, Hernández A, Prieto E. 2006. Edad y peso a la pubertad y su relación con la eficiencia reproductiva de grupos raciales bovinos en el trópico colombiano. Livestock Res Rural Develop 18: 10. http://www.lrrd.org/ lrrd18/10/graj18139.htm

9. Hare E, Norman H, Wright J. 2006. Trends in calving age and calving intervals for dairy cattle breeds in the United States. J Dairy Sci 89: 365-370.

10. Korte CJ, Chu AC, Field TR. 1987. Pasture production. In: Livestock Feeding on Pasture (Nicol AM ed). Occ. Publ. N ${ }^{\circ} 10$, N. Zealand Soc Anim Prod, Hamilton, New Zealand, p. 7-20.

11. Krupic ML, Charmandarian AR, Pagni C, Marini P. 2015. Hip height measurements, production and reproduction in adult dairy cows in Argentina grazing systems. Asian J Agric Food Sci 3: 109-112.

12. Mauren SC, Castillo G, Murillo J, Hueckmann F, Romero JJ. 2013. Edad al primer parto en vacas holstein de lechería especializada en Costa Rica. Agron Mesoam 24: 233-243.

13. Molinuevo HA. 2005. Genética bovina y producción en pastoreo, Publ. INTA (Argentina), p. 283-315.

14. Moore RK, Kennedy BW, Schaeffer LR, Moxley JE. 1991. Relationships between age and body weight at calving and production in first lactation ayrshires and holsteins. J Dairy Sci 74: 269-278.

15. Nilforooshan MA, Edriss MA. 2004. Effect of age at first calving on some productive and longevity traits in iranian holsteins of the Isfahan Province. J Dairy Sci 87: 21302135.

16. Nix JS. 1989. Economic aspects of grass production and utilization. In: Grass, its production and utilization (Holmes CW ed), Blackwell Sci. Publications, 2nd ed, London, p. 214-239. 
17. Ozawa T, López-Villalobos N, Blair HT. 2005. Dairy farming financial structures in Hokkaido, Japan and New Zealand. J Anim Sci 76: 391-400.

18. Pirlo G, Miglior F, Speroni M. 2000. Effect of age at first calving on production traits and on difference between milk yield returns and rearing costs on italian holsteins. $J$ Dairy Sci 83: 603-608.

19. Quiroz K, Carmona C, Echeverri JJ. 2011. Parámetros genéticos para algunas características productivas y reproductivas en un hato holstein del oriente antioqueño, Colombia. Rev Fac Nac Agron Medellín, 64: 2, 6199-6206.

20. Radcliff RP, VandeHaar MJ, Chapin LT et al. 2000. Effects of diet and injection of bovine somatotropin on prepubertal growth and first-lactation milk yields of holstein cows. J Dairy Sci 83: 23-29.

21. Rodriguez H, Hultgren J, Båge $\mathbf{R}$ et al. 2008. La eficiencia reproductiva en vacas lecheras de alta producción: ¿es sostenible con las prácticas de manejo actuales? Reviews in Vet Med IVIS (Publ. Internat Vet Inform Serv), Ithaca, New York, USA (www.ivis.org).
22. Salazar M, Castillo G, Murillo J, Romero JJ. 2013. Edad al primer parto en vacas holstein de lechería especializada en Costa Rica. Agron Mesoam 24: 233-243.

23. Snyder M. 2006. La recría de vaquillonas en el negocio del tambo. Producir XXI 14: 43-49.

24. Sørensen AC, Lawlor T, Ruiz F. 2007. A survey on fertility in the holstein populations of the world. Proceedings of the Int Conf Fertility Dairy Cows, Liverpool Hope University, UK, p. 30-31.

25. Vélez JS, Botero JJ, Aylor EG. 2000. Manejo reproductivo del rodeo lechero (conferencia). Actas del V Congreso Argentino de Reproducción Animal, Rosario (Santa Fe, Argentina), p. 14.

\section{Revista Veterinaria obtuvo el máximo nivel de categorización del CAICYT-CONICET}

Tras el pertinente proceso de evaluación según criterios de calidad editorial, en setiembre de 2005 CAICYT-CONICET ha clasificado a nuestra publicación con Categoría 1 (nivel superior de excelencia), con lo cual pasa a integrar el Catálogo Latindex (folio 14022). La Dirección de Revista veterinaria agradece a quienes colaboraron para obtener tan importante distinción. Ver: http://www.latindex. unam.mx/busquedas/catalogotitulo.html 